\title{
Aumann Type Set-valued Lebesgue Integral and Representation Theorem
}

\author{
Jungang Li, Shoumei Li \\ Department of Applied Mathematics, Beijing University of Technology, \\ 100 Pingleyuan, Chaoyang District, \\ Beijing, 100124, P.R.China \\ E-mail: jungangl@yahoo.cn, lisma@bjut.edu.cn \\ Received: 31-05-2008 \\ Revised: 29-01-2009
}

\begin{abstract}
In this paper, we shall firstly illustrate why we should discuss the Aumann type set-valued Lebesgue integral of a set-valued stochastic process with respect to time $t$ under the condition that the set-valued stochastic process takes nonempty compact subset of $d$-dimensional Euclidean space. After recalling some basic results about set-valued stochastic processes, we shall secondly prove that the Aumann type set-valued Lebesgue integral of a set-valued stochastic process above is a set-valued stochastic process. Finally we shall give the representation theorem, and prove an important inequality of the Aumann type set-valued Lebesgue integrals of set-valued stochastic processes with respect to $t$, which are useful to study set-valued stochastic differential inclusions with applications in finance.
\end{abstract}

Keywords: set-valued stochastic process, set-valued Lebesgue integral, Aumann type integral, representation theorem.

\section{Introduction}

In studying the evolution of macro-systems in economic, social or biological sciences, the dynamical systems having velocities are not determined uniquely by the state of systems. Thus, we study the differential inclusion instead of differential equation. A stochastic differential inclusion is defined as

$$
d x_{t} \in F_{t}\left(x_{t}\right) d t+G_{t}\left(x_{t}\right) d B_{t}, \quad x_{0}=\xi,
$$

which can be written in stochastic integral form as

$x_{t}-x_{s} \in \mathrm{cl}_{L^{2}}\left(\int_{s}^{t} F_{\tau}\left(x_{\tau}\right) d \tau+G_{\tau}\left(x_{\tau}\right) d B_{\tau}\right), \quad s, t \in I$,

where $F, G$ are set-valued stochastic processes, $B=\left(B_{t}\right)_{t \in I}$ is a Brownian motion, $\int_{s}^{t} F_{\tau}\left(x_{\tau}\right) d \tau$ is the Aumann type Lebesgue integral of the setvalued stochastic process $F$ with respect to time $\tau, \int_{s}^{t} G_{\tau}\left(x_{\tau}\right) d B_{\tau}$ is the Aumann type Ito integral of the set-valued stochastic process $G$ with respect to the Brownian motion $B$. It appears in many problems, for instance, it can be considered in a natural way as a theoretical description of stochastic control problems ${ }^{1}$.

There are many related former works about set-valued Lebesgue integral. Based on the work of Richter ${ }^{2}$ and $\mathrm{Kudo}^{3}$, Aumann ${ }^{4}$ introduced the Lebesgue integral of a set-valued function and discussed its properties. Kisielewicz ${ }^{5}$ introduced the Aumann type Lebesgue integral of a set-valued stochastic process. Kisielewicz with his colleagues ${ }^{1}$ ${ }^{5}{ }^{9}$ did a lot of nice works about stochastic differ- 
ential inclusions, especially they discussed solution problems. We naturally expect that the Aumann type Lebesgue integral of a set-valued stochastic process is a set-valued stochastic process, which is useful for applications. If $F$ takes nonempty closed set-values, we can not prove it directly, but by taking decomposable closure. $\mathrm{Li}$ and $\mathrm{Li}^{10}$ gave the definition of the Lebesgue integral of a set-valued stochastic process by decomposable closure and discussed more properties of the integral. We also would like to refer to related works such as Ref.11-14 and so on.

However, a set-valued stochastic process usually takes compact subset of $R^{d}$ space ( $d$-dimensional Euclidean space) in the real world. For example, in the famous Black-Scholes formula for the price of a European call option, the stock price $s_{t}$ at the time $t$ is assumed to satisfy

$$
d s_{t}=s_{t}\left(u d t+v d B_{t}\right)
$$

where $s_{0}>0, u, v$ are constants, $u$ is the drift of stock, $v$ is the volatility of stock and $B_{t}$ is a Brownian motion. However, being incomplete or vague of information, one usually predicts the drift of stock within some bounded interval, for example, $\left[u_{1}, u_{2}\right]$, $u_{1}<u_{2}$, rather than an exact number or an unbounded interval, for the unbounded interval usually has no actual sense. Similarly for the volatility of stock. This becomes a set-valued stochastic differential inclusion as follows:

$$
d s_{t} \in s_{t}\left(U_{t} d t+V_{t} d B_{t}\right),
$$

where $U_{t}, V_{t}$ are set-valued stochastic processes taking compact subsets of $R$ as their values. Under the condition that a set-valued stochastic process takes nonempty compact subset of $R^{d}$, can we prove that the Aumann type set-valued Lebesgue integral is a set-valued stochastic process? What properties does the integral have? These are the problems we shall solve in this paper. Fortunately, we also find an almost everywhere problem in the former definition of set-valued Aumann type Lebesgue integral, and shall solve it.

We organize our paper as following: in Section 2 , we shall introduce some necessary notations, definitions and results about set-valued stochastic processes. In Section 3, we shall discuss the former definition of Aumann type set-valued Lebesgue integral and prove that the Aumann type Lebesgue integral is a set-valued stochastic process and other properties of the integral, especially representation theorem of this type integral and an important inequality, which are useful in the study of set-valued stochastic differential inclusions. Finally we shall give an example for its application in Finance and show conclusions and acknowledgement.

\section{Set-valued Stochastic Processes}

Throughout this paper, assume that $(\Omega, \mathscr{A}, \mu)$ is a complete atomless probability space, the $\sigma$-field filtration $\left\{\mathscr{A}_{t}: t \in I\right\}$ satisfies the usual conditions (i.e. containing all null sets, non-decreasing and right continuous). $R$ is the set of all real numbers, $N$ is the set of all natural numbers, $R^{d}$ is the $d$-dimensional Euclidean space with usual norm $\|\cdot\|, \mathscr{B}(E)$ is the Borel field of the metric space $E$. Let $f=\left\{f(t), \mathscr{A}_{t}\right.$ : $t \in I\}$ be a $R^{d}$-valued adapted stochastic process. It is said that $f$ is progressively measurable if for any $t \in I$, the mapping $(s, \omega) \mapsto f(s, \omega)$ from $[0, t] \times \Omega$ to $R^{d}$ is $\mathscr{B}([0, t]) \times \mathscr{A}_{t}$-measurable.

Each right continuous (left continuous) adapted process is progressively measurable.

Assume that $\mathscr{L}^{p}\left(R^{d}\right)$ denotes the set of $R^{d}$ valued stochastic processes $f=\left\{f(t), \mathscr{A}_{t}: t \in I\right\}$ such that $f$ satisfying (i) $f$ is progressively measurable; and (ii)

$$
\||f|\|_{p}=\left[E\left(\int_{0}^{T}\|f(t, \omega)\|^{p} d s\right)\right]^{1 / p}<\infty .
$$

Let $f, f^{\prime} \in \mathscr{L}^{p}\left(R^{d}\right), f=f^{\prime}$ if and only if $\| \mid f-$ $f^{\prime} \mid \|_{p}=0$. Then $\left(\mathscr{L}^{p}\left(R^{d}\right),\|\| \cdot\|\|_{p}\right)$ is complete.

Now we review notation and concepts of setvalued stochastic processes.

Assume that $\mathbf{K}\left(R^{d}\right)$ is the family of all nonempty, closed subsets of $R^{d}$, and $\mathbf{K}_{c}\left(R^{d}\right)$ (resp. $\mathbf{K}_{k}\left(R^{d}\right)$, $\mathbf{K}_{k c}\left(R^{d}\right)$ ) is the family of all nonempty closed convex (resp. compact, compact convex) subsets of $R^{d}$. For any $x \in R^{d}, A$ is a nonempty subset of $R^{d}$, define the distance of $x$ and $A$ as $d(x, A)=\inf _{y \in A}\|x-y\|$. The Hausdorff metric on $\mathbf{K}\left(R^{d}\right)$ is defined as

$$
d_{H}(A, B)=\max \left\{\sup _{a \in A} d(a, B), \sup _{b \in B} d(b, A)\right\}
$$


for $A, B \in \mathbf{K}\left(R^{d}\right)$. For $B \in \mathbf{K}\left(R^{d}\right)$, define $\|B\|_{\mathbf{K}}=$ $d_{H}(\{0\}, B)=\sup _{a \in B}\|a\|$.

For a set-valued random variable $F$ (Ref. 15,16$)$, define the set

$S_{F}^{p}=\left\{f \in L^{p}\left[\Omega ; R^{d}\right]: f(\omega) \in F(\omega)\right.$ for a.e. $\left.\omega \in \Omega\right\}$,

where $L^{p}\left[\Omega ; R^{d}\right]$ is the set of all $R^{d}$-valued random variables $f$ such that $\|f\|_{p}=\left[E\left(\|f\|^{p}\right)\right]^{1 / p}<\infty$, and constant $p \geqslant 1$. The expectation of $F$ is defined as $E[F]=\left\{E[f]: f \in S_{F}^{1}\right\}$. It is called Aumann integral introduced by Aumann ${ }^{4}$ in 1965 . A setvalued random variable $F: \Omega \rightarrow \mathbf{K}\left(R^{d}\right)$ is called integrable if $S_{F}^{1}$ is non-empty. $F$ is called integrable bounded if $\int_{\Omega}\|F(\omega)\|_{\mathbf{K}} d \mu<\infty$. Let $L^{p}\left[\Omega ; \mathbf{K}\left(R^{d}\right)\right]$ (resp. $L^{p}\left[\Omega ; \mathbf{K}_{c}\left(R^{d}\right)\right], L^{p}\left[\Omega ; \mathbf{K}_{k c}\left(R^{d}\right)\right]$ ) denote the family of $\mathbf{K}\left(R^{d}\right)$-valued (resp. $\mathbf{K}_{c}\left(R^{d}\right), \mathbf{K}_{k c}\left(R^{d}\right)$ valued) $L^{p}$-bounded random variables $F$ such that $\|F(\cdot)\|_{\mathbf{K}} \in L^{p}[\Omega ; R]$. For any two set-valued random variables $F_{1}, F_{2} \in L^{p}\left[\Omega ; \mathbf{K}\left(R^{d}\right)\right]$, define

$$
\Delta_{p}\left(F_{1}, F_{2}\right)=\left(\int_{\Omega} d_{H}^{p}\left(F_{1}(\omega), F_{2}(\omega)\right) d \mu\right)^{1 / p}
$$

then $\left(L^{p}\left[\Omega ; \mathbf{K}\left(R^{d}\right)\right], \Delta_{p}\right)$ is a complete space. Concerning more definitions and more results of setvalued random variables, readers could refer to the excellent paper ${ }^{15}$ or the book ${ }^{16}$.

Definition 1. A set-valued stochastic process $F=$ $\{F(t): t \in I\}$ is called progressively measurable, if for any $A \in \mathscr{B}\left(R^{d}\right)$ and any $t \in I, \quad\{(s, \omega) \in$ $[0, t] \times \Omega: F(s, \omega) \cap A \neq \emptyset\} \in \mathscr{B}([0, t]) \times \mathscr{A}_{t} . \quad F$ is called $\mathscr{L}^{p}$-bounded, if the real stochastic process $\left\{\|F(t)\|_{\mathbf{K}}, \mathscr{A}_{t}: t \in I\right\} \in \mathscr{L}^{p}(R)$.

Definition 2. A $R^{d}$-valued stochastic process $\left\{f(t), \mathscr{A}_{t}: t \in I\right\} \in \mathscr{L}^{p}\left(R^{d}\right)$ is called an $\mathscr{L}^{p}$ selection of $F=\left\{F(t), \mathscr{A}_{t}: t \in I\right\}$ if $f(t, \omega) \in$ $F(t, \omega)$ for a.e. $(t, \omega) \in I \times \Omega$.

Let $S^{p}(\{F(\cdot)\})$ or $S^{p}(F)$ denote the family of all $\mathscr{L}^{p}$-selections of $F=\left\{F(t), \mathscr{A}_{t}: t \in I\right\}$, i.e.

$$
\begin{aligned}
S^{p}(F)=\{ & \{f(t): t \in I\} \in \mathscr{L}^{p}\left(R^{d}\right): f(t, \omega) \in \\
& F(t, \omega), \text { for a.e. }(t, \omega) \in I \times \Omega\} .
\end{aligned}
$$

Let $\mathscr{L}^{p}\left(\mathbf{K}\left(R^{d}\right)\right)$ denote the set of all $\mathscr{L}^{p}$ bounded progressively measurable $\mathbf{K}\left(R^{d}\right)$-valued stochastic processes. Similarly, we have notations $\mathscr{L}^{p}\left(\mathbf{K}_{c}\left(R^{d}\right)\right), \quad \mathscr{L}^{p}\left(\mathbf{K}_{k}\left(R^{d}\right)\right)$ and $\mathscr{L}^{p}\left(\mathbf{K}_{k c}\left(R^{d}\right)\right)$. Take $F_{i}=\left\{F_{i}(t): t \in I\right\} \in \mathscr{L}^{p}\left(\mathbf{K}\left(R^{d}\right)\right), i=1,2$, define

$\boldsymbol{\Delta}_{p}\left(F_{1}, F_{2}\right)=\left[E\left(\int_{0}^{T} d_{H}^{p}\left(F_{1}(s, \omega), F_{2}(s, \omega)\right) d s\right)\right]^{1 / p}$.

$F_{1}$ and $F_{2}$ are said to be equivalent, if $\boldsymbol{\Delta}_{p}\left(F_{1}, F_{2}\right)=0$, denoted by $F_{1}=F_{2}$. We have that $\left(\mathscr{L}^{p}\left(\mathbf{K}\left(R^{d}\right)\right), \mathbf{\Lambda}_{p}\right)$ is complete, $\mathscr{L}^{p}\left(\mathbf{K}_{c}\left(R^{d}\right)\right)$, $\mathscr{L}^{p}\left(\mathbf{K}_{k}\left(R^{d}\right)\right)$ and $\mathscr{L}^{p}\left(\mathbf{K}_{k c}\left(R^{d}\right)\right)$ are closed subsets of $\left(\mathscr{L}^{p}\left(\mathbf{K}\left(R^{d}\right)\right), \mathbf{\Delta}_{p}\right)$. Denote $\|\mid F\|_{p}=$ $\left[E\left(\int_{0}^{T}\|F(s)\|_{\mathbf{K}}^{p} d s\right)\right]^{1 / p}$.

Theorem 1. Let $F \in \mathscr{L}^{p}\left(\mathbf{K}\left(R^{d}\right)\right)$ with $p \geqslant 1$, then $S^{1}(F)=S^{p}(F)$.

Proof. $\quad S^{p}(F) \subseteq S^{1}(F)$ is obvious. Now we prove the converse. For any $f \in S^{1}(F)$, we have $\|f(s, \omega)\| \leqslant\|F(s, \omega)\|_{\mathbf{K}}$ since $f(s, \omega) \in F(s, \omega)$ for a.e. $(t, \omega) \in I \times \Omega$. Note that $F \in \mathscr{L}^{p}\left(\mathbf{K}\left(R^{d}\right)\right)$, so that we have $f \in \mathscr{L}^{p}\left(R^{d}\right)$, which implies $S^{1}(F) \subseteq$ $S^{p}(F)$.

\section{Aumann Type Set-valued Lebesgue Integral and its Properties}

Now we give the definition of Aumann type Lebesgue integral of a set-valued stochastic process with respect to time $t$.

Definition 3. Let a set-valued stochastic process $F=\{F(t): t \in I\} \in \mathscr{L}^{p}\left(\mathbf{K}\left(R^{d}\right)\right)(1 \leqslant p<+\infty)$. For any $t \in I, \omega \in \Omega$, define

$$
\text { (A) } \int_{0}^{t} F(s, \omega) d s:=\left\{\int_{0}^{t} f(s, \omega) d s: f \in S^{p}(F)\right\},
$$

where $\int_{0}^{t} f(s, \omega) d s$ is the Lebesgue integral. (A) $\int_{0}^{t} F(s, \omega) d s$ is called the Aumann type Lebesgue integral of the set-valued stochastic process $F$ with respect to time $t$ introduced in Ref.5. For any $0 \leqslant u<t<T$,

$$
\text { (A) } \int_{u}^{t} F(s, \omega) d s:=(A) \int_{0}^{t} I_{[u, t]}(s) F(s, \omega) d s .
$$

Remark 1. (1) In the definition 3, the set of selections is $S^{p}(F)$. As a matter of fact, if we only 
consider the Lebesgue integral, we can use $S^{1}(F)$. But we often consider the sum of the Lebesgue integral of a set-valued stochastic process with respect to time $t$ and the Ito integral of a set-valued stochastic process with respect to the Brownian motion, where we have to use $S^{2}(F)$. Thus we here use $S^{p}(F)$ for more general case.

(2) There is a delicate problem in the definition above, i.e. is the Aumann type Lebesgue integral of a stochastic process well-defined for every $\omega \in \Omega$ ? As matter of fact, take an $f \in S^{p}(F)$. Then, for any fixed $t \in I$, by Fubini Theorem, the mapping $f(\cdot, \omega):[0, t] \rightarrow R^{d}$ is $\mathscr{B}([0, t])$-measurable for all $\omega \in \Omega$, but ONLY for a.e. $\omega \in \Omega$ (NOT every $\omega \in \Omega$ !),

$$
I_{t}(f)(\omega)=\int_{0}^{t} f(s, \omega) d s<\infty .
$$

Now the problem appears: $I_{t}(f)$ is defined a.e. $\omega \in$ $\Omega$ for each $f$ and the set $\left\{I_{t}(f): f \in S^{p}(F)\right\}$ is usually uncountable. We should notice that the union of the exceptional sets may not be of measure zero. If not, $I_{t}(F)$ is not well-defined even for a.e. $\omega \in \Omega$ ! Under what kind of condition, is the above $I_{t}(F)$ well-defined for a.e. $\omega \in \Omega$ ?

To solve the problem, we assume that $\mathscr{A}$ is $\mu$ separable in this paper. In this case, we have that $S^{p}(F)$ is separable (Ref.17). Thus the Aumann type Lebesgue integral $I_{t}(F)$ is well-defined for a.e. $\omega \in \Omega$. Without loss of generalization, we assume that for every $\omega \in \Omega$, definition 3 and the following hold.

Now we prove that the Aumann type set-valued Lebesgue integral is a stochastic process.

Theorem 2. Assume that a set-valued stochastic process $F \in \mathscr{L}^{p}\left(\mathbf{K}_{k}\left(R^{d}\right)\right)$. Then the set-valued mapping $L_{t}(F): \Omega \rightarrow \mathbf{K}_{k c}\left(R^{d}\right)$ defined by

$$
L_{t}(F)(\omega)=(A) \int_{0}^{t} F(s, \omega) d s
$$

is measurable, i.e. $L_{t}(F)$ is a set-valued random variable, and

$$
L_{t}(F)(\omega)=(A) \int_{0}^{t} \operatorname{co} F(s, \omega) d s
$$

Proof. When $p=1$, we have

$$
\begin{aligned}
L_{t}(F)(\omega) & =(A) \int_{0}^{t} F(s, \omega) d s \\
& =\left\{\int_{0}^{t} f(s, \omega) d s: f \in S^{1}(F)\right\} .
\end{aligned}
$$

From Theorem II.3.20 in Ref.6, (A) $\int_{0}^{t} F(s, \omega) d s$ takes nonempty compact and convex subsets of $R^{d}$ as its values and we have

$$
\text { (A) } \int_{0}^{t} F(s, \omega) d s=(A) \int_{0}^{t} \operatorname{co} F(s, \omega) d s .
$$

Since $F: I \times \Omega \rightarrow \mathbf{K}_{k}\left(R^{d}\right)$ is progressively measurable, by Remark II.3.5 in Ref.6, $I \times \Omega \ni(t, \omega) \rightarrow$ $\sigma(x, F(t, \omega)) \in R$ is measurable for every $x \in R^{d}$, where $\sigma(x, A)=\sup \{\langle x, y\rangle: y \in A\}$ for $A \subset R^{d}$. By virtue of Theorem II.3.21 in Ref.6, we have

$$
\int_{0}^{t} \sigma(x, F(s, \omega)) d s=\sigma\left(x,(A) \int_{0}^{t} F(s, \omega) d s\right)
$$

for every $x \in R^{d}, \omega \in \Omega$. So $L_{t}(F)(\omega)=$ (A) $\int_{0}^{t} F(s, \omega) d s$ is measurable by Theorem II.3.8 in Ref.6 or Proposition I.2.5 in Ref.18. Thus (A) $\int_{0}^{t} F(s, \omega) d s$ is measurable when $p=1$. Since $F \in \mathscr{L}^{p}\left(\mathbf{K}_{k}\left(R^{d}\right)\right)$, we have $S^{1}(F)=S^{p}(F)$ by Theorem 1. So $L_{t}(F)(\omega)=(A) \int_{0}^{t} F(s, \omega) d s$ is measurable with respect to $\omega \in \Omega$ for any $p \geqslant 1$.

Remark 2. (1) The Aumann type set-valued Lebesgue integral defined in Theorem 2 is a set-valued stochastic process denoted by $L(F)=$ $\left\{L_{t}(F): t \in I\right\}$. Please notice that we proved it under the condition of the set-valued stochastic process $F$ taking nonempty COMPACT set values.

(2) We are interested in the set of all selections of the integral stochastic process $L(F)$. For any fixed $t \in I$, by Fubini Theorem, $I_{t}(f)(\omega)=: \int_{0}^{t} f(s, \omega) d s$ is an $\mathscr{A}_{t}$-measurable function with respect to $\omega$ for any given $f \in S^{p}(F)$. Thus $I_{t}(f)(\cdot)=: \int_{0}^{t} f(s, \cdot) d s$ is a selection of $L_{t}(F)$. By the classical Jensen inequality, we have $I_{t}(f) \in L^{p}\left[\Omega, \mathscr{A}_{t}, \mu ; R^{d}\right]$. Thus, $\left\{I_{t}(f): f \in\right.$ $\left.S^{p}(F)\right\}$ is a non-empty subset of $L^{p}\left[\Omega, \mathscr{A}_{t}, \mu ; R^{d}\right]$. As a matter of fact, we have the following Theorem.

Theorem 3. Assume that a set-valued stochastic process $F \in \mathscr{L}^{p}\left(\mathbf{K}_{k}\left(R^{d}\right)\right)$ and continue to use above notations. Then we have that $\left\{I_{t}(f): f \in S^{p}(F)\right\}$ is closed in $L^{p}\left[\Omega, \mathscr{A}_{t}, \mu ; R^{d}\right]$. 
Proof. Take a sequence $\left\{\left\{f_{n}(t): t \in I\right\}: n \in N\right\} \subset$ $S^{p}(F)$ such that $\left\{\phi_{n}(t): n \in N\right\}=:\left\{\int_{0}^{t} f_{n}(s) d s: n \in\right.$ $N\} \subset\left\{I_{t}(f): f \in S^{p}(F)\right\}$ is a Cauchy sequence in $L^{p}\left[\Omega, \mathscr{A}_{t}, \mu ; R^{d}\right]$. Since $L^{p}\left[\Omega, \mathscr{A}_{t}, \mu ; R^{d}\right]$ is complete, there exists $\phi(t) \in L^{p}\left[\Omega, \mathscr{A}_{t}, \mu ; R^{d}\right]$ such that

$$
\begin{aligned}
& E\left[\left\|\phi_{n}(t)-\phi(t)\right\|^{p}\right] \\
& =E\left[\left\|\int_{0}^{t} f_{n}(s) d s-\phi(t)\right\|^{p}\right] \\
& \rightarrow 0, \quad(n \rightarrow \infty) .
\end{aligned}
$$

Thus, there exists a subsequence $\left\{f_{n_{k}}: k \in N\right\}$ of $\left\{f_{n}: n \in N\right\}$ such that when $k \rightarrow \infty$, we have

$$
\int_{0}^{t} f_{n_{k}}(s, \omega) d s \rightarrow \phi(t, \omega), \text { a.e. } \omega \in \Omega .
$$

By Theorem 2, $L_{t}(F)(\omega)$ is a compact subset of $R^{d}$. This, with the fact $I_{t}\left(f_{n_{k}}\right)(\omega)=\int_{0}^{t} f_{n_{k}}(s, \omega) d s \in$ $L_{t}(F)(\omega)$, implies

$$
\phi(t, \omega) \in L_{t}(F)(\omega) \text {, a.e. } \omega \in \Omega .
$$

Therefore, $\left\{I_{t}(f): f \in S^{p}(F)\right\}$ is a closed subset of $L^{p}\left[\Omega, \mathscr{A}_{t}, \mu ; R^{d}\right]$.

On the other hand, from Theorem 2, we know that $\left\{I_{t}(f): f \in S^{p}(F)\right\}$ is decomposable (Ref.15). Therefore, $S_{L_{t}(F)}^{1}\left(\mathscr{A}_{t}\right)=\left\{I_{t}(f): f \in S^{p}(F)\right\}$ from Theorem 3.

The following Lemma and Theorem are about representation theorem of the Aumann type setvalued Lebesgue integral.

Lemma 4. If the set-valued stochastic process $F \in$ $\mathscr{L}^{p}\left(\mathbf{K}_{k}\left(R^{d}\right)\right)$, then there exists a sequence $\left\{f^{n}: n \in\right.$ $N\} \subset S^{p}(F)$ such that for any $t \in I$,

$$
S_{L_{t}(F)}^{1}\left(\mathscr{A}_{t}\right)=\operatorname{cl}\left\{\int_{0}^{t} f^{n}(s) d s: n \in N\right\},
$$

where the closure is taken in $L^{1}$.

Proof. Since we assume that $\mathscr{A}$ is $\mu$-separable, we have that $\mathscr{L}^{p}\left(R^{d}\right)$ is separable (Ref.17). Thus $S^{p}(F)$ is also separable since it is a closed subset of $\mathscr{L}^{p}\left(R^{d}\right)$ (Theorem 2.4 in Ref.10). That is, there exists a sequence $\left\{f^{n}: n \in N\right\} \subset S^{p}(F)$ such that $S^{p}(F)=\operatorname{cl}\left\{f^{n}: n \in N\right\}$, where the closure is taken in $\mathscr{L}^{p}\left(R^{d}\right)$.
For any $t \in I, S_{L_{t}(F)}^{1}\left(\mathscr{A}_{t}\right)=\left\{\int_{0}^{t} f(s) d s: f \in\right.$ $\left.S^{p}(F)\right\}$. We only need to prove

$\left\{\int_{0}^{t} f(s) d s: f \in S^{p}(F)\right\} \subset \operatorname{cl}\left\{\int_{0}^{t} f^{n}(s) d s: n \in N\right\}$,

where the closure is taken in $L^{1}$, since the opposite inclusion is obvious.

Let $g \in S^{p}(F)$, then there exists a subsequence $\left\{f^{n_{i}}: i \geqslant 1\right\}$ of $\left\{f^{n}: n \in N\right\}$ such that $\left(E \int_{0}^{T}\left\|g(t, \omega)-f^{n_{i}}(t, \omega)\right\|^{p} d t\right)^{\frac{1}{p}} \rightarrow 0(i \rightarrow \infty)$. We know that $\int_{0}^{t} g(s) d s \in\left\{\int_{0}^{t} f(s) d s: f \in S^{p}(F)\right\}$, and

$$
\begin{aligned}
& E\left\|\int_{0}^{t} g(s, \omega) d s-\int_{0}^{t} f^{n_{i}}(s, \omega) d s\right\| \\
& \leqslant E \int_{0}^{t}\left\|g(s, \omega)-f^{n_{i}}(s, \omega)\right\| d s \\
& \leqslant E \int_{0}^{T}\left\|g(s, \omega)-f^{n_{i}}(s, \omega)\right\| d s \\
& \rightarrow 0 \quad(i \rightarrow 0),
\end{aligned}
$$

which means $\int_{0}^{t} g(s) d s \in \operatorname{cl}\left\{\int_{0}^{t} f^{n}(s) d s: n \in N\right\}$. Therefore,

$\left\{\int_{0}^{t} f(s) d s: f \in S^{p}(F)\right\} \subset \operatorname{cl}\left\{\int_{0}^{t} f^{n}(s) d s: n \in N\right\}$.

The proof is completed.

Theorem 5. (Representation Theorem) For any set-valued stochastic process $F \in \mathscr{L}^{p}\left(\mathbf{K}_{k}\left(R^{d}\right)\right)$, there exists a sequence $\left\{f^{i}=\left\{f^{i}(t, \omega): t \in I, \omega \in\right.\right.$ $\Omega\}: i \in N\} \subset S^{p}(F)$ such that for a.e. $(t, \omega) \in I \times \Omega$,

$$
F(t, \omega)=\operatorname{cl}\left\{f^{i}(t, \omega): i \in N\right\},
$$

and

$$
L_{t}(F)(\omega)=\operatorname{cl}\left\{\int_{0}^{t} f^{i}(s, \omega) d s: i \in N\right\} .
$$

Proof. For $F \in \mathscr{L}^{p}\left(\mathbf{K}_{k}\left(R^{d}\right)\right)$, by Lemma 4 , there exists a sequence $\left\{h^{n}=\left\{h^{n}(t): t \in I\right\}: n \in N\right\} \subset$ $S^{p}(F)$ such that for any $t \in I$,

$$
S_{L_{t}(F)}^{1}\left(\mathscr{A}_{t}\right)=\operatorname{cl}\left\{\int_{0}^{t} h^{n}(s) d s: n \in N\right\},
$$

where the closure is taken in $L^{1}$. By Theorem 1.3.1 in Ref.16, for any $\omega \in \Omega, L_{t}(F)(\omega)=$ $\operatorname{cl}\left\{\int_{0}^{t} h^{n}(s, \omega) d s: n \in N\right\}$. Due to Theorem 2.5 in 
Ref.10, there exits a sequence $\left\{\phi^{j}=\left\{\phi^{j}(t): t \in I\right\}\right.$ : $j \in N\} \subset S^{p}(F)$ such that for any $(t, \omega) \in I \times \Omega$,

$$
F(t, \omega)=\operatorname{cl}\left\{\phi^{j}(t, \omega): j \in N\right\} .
$$

Take the element one by one from two sequences $\left\{h_{n}: n \in N\right\},\left\{\phi^{j}: j \in N\right\}$, and get a new sequence $\left\{h^{1}, \phi^{1}, h^{2}, \phi^{2}, \cdots\right\}$, and denoted as

$$
\left\{f^{i}=\left\{f^{i}(t, \omega): t \in I, \omega \in \Omega\right\}: i \in N\right\},
$$

then for any $(t, \omega) \in I \times \Omega$,

$$
F(t, \omega) \subset \operatorname{cl}\left\{f^{i}(t, \omega): i \in N\right\} .
$$

By the definition of selection, for each $n \in N$ and a.e. $(t, \omega) \in I \times \Omega, h^{n}(t, \omega) \in F(t, \omega)$. Thus for a.e. $(t, \omega) \in I \times \Omega$,

$$
\operatorname{cl}\left\{f^{i}(t, \omega): i \in N\right\} \subset F(t, \omega) .
$$

Therefore, for a.e. $(t, \omega) \in I \times \Omega$,

$$
F(t, \omega)=\operatorname{cl}\left\{f^{i}(t, \omega): i \in N\right\} .
$$

In addition, for each $j \in N$ and a.e. $(t, \omega) \in I \times \Omega$,

$$
\int_{0}^{t} \phi^{j}(s, \omega) d s \in L_{t}(F)(\omega)
$$

then

$$
\operatorname{cl}\left\{\int_{0}^{t} \phi^{j}(s, \omega) d s: j \in N\right\} \subset L_{t}(F)(\omega) .
$$

Therefore, for a.e. $(t, \omega) \in I \times \Omega$,

$$
L_{t}(F)(\omega)=\operatorname{cl}\left\{\int_{0}^{t} f^{i}(s, \omega) d s: i \in N\right\} .
$$

The proof is completed.

Now we prove an inequality of set-valued Legesgue integrals.

Theorem 6. Let $p \geqslant 1$. Then, for any $F, G \in$ $\mathscr{L}^{p}\left(\mathbf{K}_{k}\left(R^{d}\right)\right)$, we have

$$
\begin{aligned}
& d_{H}^{2}\left(L_{t}(F)(\omega), L_{t}(G)(\omega)\right) \\
& \leqslant t \int_{0}^{t} d_{H}^{2}(F(s, \omega), G(s, \omega)) d s .
\end{aligned}
$$

Proof. For each $f \in S^{p}(F)$ and for any $\omega \in \Omega$, we have

$$
\begin{aligned}
& \inf _{y \in L_{t}(G)(\omega)}\left\|I_{t}(f)(\omega)-y\right\|^{2} \\
& \quad=\inf _{g \in S^{p}(G)}\left\|\int_{0}^{t} f(s, \omega) d s-\int_{0}^{t} g(s, \omega) d s\right\|^{2} \\
& \leqslant t \inf _{g \in S^{p}(G)} \int_{0}^{t}\|f(s, \omega)-g(s, \omega)\|^{2} d s .
\end{aligned}
$$

By Lemma 1.3.12 in Ref.16, we have

$$
\begin{gathered}
\inf _{g \in S^{p}(G)} \int_{0}^{t}\|f(s, \omega)-g(s, \omega)\|^{2} d s \\
\quad=\int_{0}^{t} \inf _{y \in G(s, \omega)}\|f(s, \omega)-y\|^{2} d s \\
\quad \leqslant \int_{0}^{t} d_{H}^{2}(F(s, \omega), G(s, \omega)) d s .
\end{gathered}
$$

Noticing that

$$
\begin{aligned}
& \sup _{x \in L_{t}(F)(\omega)} \inf _{y \in L_{t}(G)(\omega)}\|x-y\|^{2} \\
& \quad=\sup _{f \in S^{p}(F)} \inf _{y \in L_{t}(G)(\omega)}\left\|I_{t}(f)(\omega)-y\right\|^{2},
\end{aligned}
$$

we have

$$
\begin{aligned}
& \sup _{x \in L_{t}(F)(\omega)} \inf _{y \in L_{t}(G)(\omega)}\|x-y\|^{2} \\
& \quad \leqslant t \int_{0}^{t} d_{H}^{2}(F(s, \omega), G(s, \omega)) d s .
\end{aligned}
$$

By the definition of Hausdorff metric and symmetrical property, we have (1).

Corollary 7. If $F \in \mathscr{L}^{2}\left(\mathbf{K}_{k}\left(R^{d}\right)\right)$ and $F(t)$ is continuous in $t$ with respect to the Hausdorff metric $d_{H}$, then $L_{t}(F)$ is continuous in $t$ with respect to $d_{H}$. Proof. Take $G=I_{[0, s]} F$, we have the conclusion by (1) and the classical dominated convergence theorem. 


\section{Note}

Now we come back to the Black-Scholes formula for the price of a European call option, the stock price $s_{t}$ at the time $t$ is assumed to satisfy

$$
d s_{t}=s_{t}\left(u d t+v d B_{t}\right)
$$

where $s_{0}>0, u$ is the drift of stock, $v$ is the volatility of stock and $B_{t}$ is a Brownian motion. When we predict the drift of stock fluctuates or takes in the interval $\left[u_{1}, u_{2}\right], u_{1}, u_{2} \in R$, then the stock price $s_{t}$ at the time $t$ satisfies the following set-valued stochastic differential inclusion

$$
d s_{t} \in\left[u_{1} s_{t}, u_{2} s_{t}\right] d t+v s_{t} d B_{t},
$$

or set-valued stochastic integral form

$$
s_{t}-s_{0} \in \mathrm{cl}_{L^{p}}\left(\in(L) \int_{0}^{t}\left[u_{1} s_{t}, u_{2} s_{t}\right] d t+\int_{0}^{t} v s_{t} d B_{t}\right),
$$

where the first integral is Aumann type set-valued Lebesgue integral, the second integral is classical It $\widehat{o}$ integral. If $s_{t} \in \mathscr{L}^{p}(R)$, then $\left\{F_{t}=\right.$ $\left.\left[u_{1} s_{t}, u_{2} s_{t}\right], \mathscr{A}_{t}, t \in I\right\} \in \mathscr{L}^{p}\left(\mathbf{K}_{k}(R)\right)$. If $h=\left\{h_{t}, t \in\right.$ $I\} \in \mathscr{L}(R)$ satisfying $u_{1} s_{t}(\omega) \leqslant h_{t}(\omega) \leqslant u_{2} s_{t}(\omega)$ for a.e. $(t, \omega) \in I \times \Omega$, then $h \in S^{p}(F)$ and $S^{p}(F)=$ $\left\{h=\left\{h_{t}, t \in I\right\} \in \mathscr{L}(R): u_{1} s_{t}(\omega) \leqslant h_{t}(\omega) \leqslant\right.$ $u_{2} s_{t}(\omega)$, for a.e. $\left.(t, \omega) \in I \times \Omega\right\}$. From Theorem 2 and Theorem 3, we have $S_{L_{t}(F)}^{1}=\left\{I_{t}(h): h \in\right.$ $\left.S^{p}(F)\right\}$. By representation theorem, there exists a sequence of real-valued stochastic processes $\left\{h^{i}=\right.$ $\left.\left\{h_{t}^{i}, t \in I\right\}, i \in N\right\} \subset S^{p}(F)$ such that for a.e. $\left(t_{0}, \omega\right) \in$ $I \times \Omega$,

$$
F\left(t_{0}, \omega\right)=\operatorname{cl}\left\{h^{i}\left(t_{0}, \omega\right): i \geqslant 1\right\}
$$

and

$$
\begin{aligned}
& L_{t_{0}}(F) \\
& =\operatorname{cl}\left\{\int_{0}^{t_{0}} h^{i}(t, \omega) d t: i \geqslant 1\right\} \\
& =\left[u_{1} \int_{0}^{t_{0}} s_{t}(\omega) d t, u_{2} \int_{0}^{t_{0}} s_{t}(\omega) d t\right] \\
& =\left[u_{1}, u_{2}\right] \int_{0}^{t_{0}} s_{t}(\omega) d t .
\end{aligned}
$$

Furthermore, we have the important inequality of the integral by Theorem 6 . So we can study the solution of set-valued stochastic differential inclusion (2) and its properties(Ref.5, 7). Thus, we can know the movement trajectory of stock price, which changes in certain range when we predict the drift of stock fluctuates or takes in a given interval. It is very useful for us to make decision in the process and system risk control.

\section{Conclusions and Acknowledgement}

In this paper, we firstly discuss two basic problems. 1) We prove that the Aumann type set-valued Lebesgue integral of a set-valued stochastic process is a set-valued stochastic process under the condition that the set-valued stochastic process takes none-empty compact subset of $R^{d}$. 2) We discuss whether the former definition of the Aumann type set-valued Lebesgue integral of a set-valued stochastic process with respect to the time $t$ is well-defined for all $\omega \in \Omega$. In this paper, we assume that $\mathscr{A}$ is $\mu$ separable in probability space $(\Omega, \mathscr{A}, \mu)$ so that the former definition is available.

We secondly obtain the representation theorem, and prove an important inequality of the Aumann type set-valued Lebesgue integrals of set-valued stochastic processes with respect to $t$. These results are important for developing the theory of set-valued stochastic differential inclusions, which are useful in finance and optimal control areas.

Finally we would like to thank referees for their valuable suggestions and remarks, which improve the presentation of this paper and thank M. Kisielewicz in Zielona Góra University and Yukio Ogura in Saga University for the valuable communication. This research is supported by NSFC(NO.10771010), Research fund of Beijing Educational Committee, PHR(IHLB) and 111 Talent Project Fund of BJUT, P.R.China.

1. M. Kisielewicz, "Set-valued stochastic integrals and stochastic inclusions," Stoch. Anal. Appl. 15, 783-800 (1997).

2. H. Richter, "Verallgemeinerung eines in der Statistik benötigten Satzes der Masstherie," Math. Annalen, 150, 85-90 (1963).

3. H. Kudo, "Dependent experiments and sufficient statistics," Nat. Sci. Rept. Ochanomizu Univ., Tokyo, 4, 151-163 (1954). 
4. R. Aumann, "Integrals of set-valued functions," $J$. Math. Anal. Appl., 12, 1-12 (1965).

5. M. Kisielewicz, "Set-valued stochastic integrals and stochastic inclusions," Discuss. Math., 13, 119-126 (1993).

6. M. Kisielewicz, Differential Inclusions and Optimal Control, Kluwer Academic Publishers, New York, 1991.

7. M. Kisielewicz, "Properties of solution set of stochastic inclusions," J. Appl. Math. Stoch. Anal., 6, 217-236 (1993).

8. M. Kisielewicz, M. Michta and J. Motyl, "Set-valued approach to stochastic control part I (existence and regularity properties)," Dynamic Syst. and Appl., 12, 405-432 (2003).

9. M. Kisielewicz, M. Michta and J. Motyl, "Set-valued approach to stochastic control part II (viability and semimartingale issues)," Dynamic Syst. and Appl., 12, 433-466 (2003).

10. J. Li and S. Li, "Set-valued stochastic Lebesgue integral and representation theorems," International Journal of Computational Intelligence Systems, 1, 177-187 (2008).

11. G. Da Prato and H. Frankowska,“ A stochastic Filippov theorem,” Stoch. Anal. Appl., 12, 409-426 (1994).

12. J. Motyl, "Existence of solutions of set-valued Ito equation," Bull. Acad. Pol. Sci., 46, 419-430 (1998).

13. Y. Ogura, On stochastic differential equations with set coefficients and the Black-Scholes model, in the Proceeding of the 8th International Conference on Intelligent Technologies, in Sydney, 300-304, 2007.

14. W. Zhang, S. Li, Z. Wang and Y. Gao, An Introduction of Set-Valued Stochastic Processes, Science Press, Beijing/New York, 2007.

15. F. Hiai and H. Umegaki, "Integrals, conditional expectations and martingales of multivalued functions,"
Jour. Multivar. Anal., 7, 149-182 (1977)

16. S. Li, Y. Ogura and V. Kreinovich, Limit Theorems and Applications of Set-Valued and Fuzzy SetValued Random Variables, Kluwer Academic Publishers, 2002.

17. J.A. Yan, Measure Theorey, Science Press, 2004.

18. I. Molchanov, Theory of Random Sets, Springer, 2005.

19. B.K. Kim and J.H. Kim, "Stochastic integrals of setvalued processes and fuzzy processes," J. Math. Anal. Appl., 236, 480-502 (1999).

20. J.P. Aubin and H. Frankowska, Set-Valued Analysis, Birkhauser, 1990.

21. B.D. Craven, Lebesgue Measure and Integral, Pitman, 1982.

22. C. Castaing and M. Valadier, Convex Analysis and Measurable Multifunctions, Lect. Notes in Math., 580, Springer-Verlag, Berlin, New York, 1977.

23. S. Hu and N.S. Papageorgiou, Handbook of Multivalued Analysis, Kluwer Academic Publishers, 1997.

24. E.J. Jung and J.H. Kim, "On set-valued stochastic integrals,” Stoch. Anal. Appl., 21, 401-418 (2003).

25. E. Klein and A.C. Thompson, Theory of Correspondences Including Applications to Mathematical Economics, John Wiley \& Sons, 1984.

26. S. Li, Y. Ogura, F.N. Proske and M.L. Puri, "Central limit theorems for generalized set-valued random variables," J. Math. Anal. Appl., 285, 250-263 (2003).

27. S. Li and A. Ren, "Representation theorems, setvalued and fuzzy set-valued Ito intergal," Fuzzy Sets and Syst., 158, 949-962 (2007).

28. N.S. Papageorgiou, "On the conditional expectation and convergence properties of random sets," Trans. Amer. Math. Soc., 347, 2495-2515 (1995).

29. J. Zhang, S. Li, I. Mitoma and Y. Okazaki, "On setvalued stochastic integrals in an M-type 2 Banach space," J. Math. Anal. Appl., 350, 216-233 (2009). 